\title{
Promotion of remyelination by polyclonal immunoglobulin in Theiler's virus-induced demyelination and in multiple sclerosis
}

\author{
Baziel G M van Engelen, David J Miller, Kevin D Pavelko, Otto R Hommes, \\ Moses Rodriguez
}

\begin{abstract}
Spontaneous remyelination occurs in experimental models of demyelination and in patients with multiple sclerosis, although to a limited extent. This enables the search for factors that promote remyelination. Using the Theiler's virus model of central nervous system demyelination, promotion of remyelination was observed after passive transfer of CNS-specific antiserum and transfer of CNS-specific purified immunoglobulin. Mouse polyclonal immunoglobulin from normal non-syngeneic mice, comparable with the human immunoglobulin preparation, also promotes CNS remyelination in the Theiler's virus model of multiple sclerosis. These experimental findings further bridge the gap with a pilot study that suggests clinical improvement after polyclonal immunoglobulin administration, possibly due to remyelination, in some multiple sclerosis patients with stable, steroid-unresponsive optic neuritis. In view of these experimental and clinical data, the physiological role of myelin in demyelination and remyelination is discussed, and the role of IgG solely as a deleterious molecule in the pathophysiology of multiple sclerosis and experimental demyelination is addressed.
\end{abstract}

Disability in multiple sclerosis (MS) results from a focal inflammatory and demyelinating process and the subsequent failure of full repair. Accordingly, treatments for MS may aim to: (1) limit inflammation and demyelination; (2) improve conduction in demyelinated fibres; (3) enhance myelin repair, that is, remyelination, and (4) prevent secondary axonopathy, that is, axon loss due to demyelination. Current treatment strategies in MS have focussed almost exclusively on number 1 , the initial stage of the disease, by tending to limit inflammation and demyelination. In our paper we focus mainly on strategy number 3, a novel treatment approach to enhance myelin repair by factors of the humoral immune system, especially exogenous immunoglobulin (IVIg).*

*For reasons of convenience the abbreviation IVIg will be used for immunoglobulin administered intravenously and intramuscularly.
Until recently, only a few studies testing IVIg treatment in MS were available, although the first reports were published in $1963 .^{23}$ This may be due to the conventional wisdom that assumes that immunoglobulins play a pathogenic role in the attack on myelin, based almost exclusively on increased intrathecal immunoglobulin production being a hallmark in the diagnosis of MS. Recently, there has been a renewed interest in IVIg treatment in MS. ${ }^{4-9}$ All except one of these studies are uncontrolled, and suggest a possible benefit in preventing and limiting the initial damage in MS. In contrast to slowing disease progression, one report focussed on the potential role of IVIg in clinical improvement possibly due to myelin repair. ${ }^{8}$

\section{Remyelination}

The traditional concept that repair in the CNS occurs rarely, if ever, seems outdated, at least for myelin. Spontaneous remyelination has now been described in toxin-induced, autoimmune, and viral-mediated models of CNS demyelination, ${ }^{10}$ and in humans with MS. ${ }^{11-13}$ Accordingly, a CNS demyelinating disease like MS is no longer conceived simply as a static and irreversible condition of myelin loss, ${ }^{14}$ but as a dynamic equilibrium between demyelinating and remyelinating factors. This enables the search for factors that can improve the incomplete process of spontaneous remyelination in MS, and that can shift the equilibrium toward remyelination.

Immunoglobulin, IVIg and remyelination Although antibodies can induce demyelination in vitro ${ }^{15}$ as well as in vivo, ${ }^{16}$ they have also been shown to promote remyelination in experimental demyelination. Antibody-mediated oligodendrocyte stimulation has been reported in vitro. ${ }^{1718}$ Analogous in vivo treatment experiments were carried out in the Theiler's murine encephalomyelitis virus (TMEV) model of demyelination. TMEV, a picornavirus, causes a chronic progressive immune-mediated CNS demyelinating disease similar pathologically and clinically to the chronic progressive form of MS. These experiments showed that passive transfer of CNS-specific antiserum, ${ }^{19}$ and purified immunoglobulin ${ }^{2021}$ promoted CNS remyelination. In contrast to the classic view of the humoral immune system playing a pathogenic role in CNS demyelination, these experiments 
indicated for the first time that immunoglobulins, and in particular autoreactive immunoglobulins, that is, autoantibodies, might play a beneficial role by promoting CNS remyelination.

In view of this effect of antibodies mediating remyelination in an established experimental model of MS, we recently published an unblinded study testing IVIg treatment in patients with non-recovering, steroid-unresponsive optic neuritis. ${ }^{8}$ In this open label study of five MS patients with non-recovering, static, optic neuritis (eight eyes; optic neuritis duration seven months to four years), the repeated administration of IVIg was followed within one or two months by improvement of visual acuity and colour vision (five eyes), light brightness (three eyes), and visual evoked responses (five eyes). Improvement was temporally related to the administration of IVIg and has persisted for the follow up period of 1.2 to 1.7 years. These findings suggest that IVIg administration in patients with non-recovering, steroid-unresponsive optic neuritis due to MS is associated with improvement of neurological function. The clinical improvement may have been due to IVIg-mediated promotion of remyelination, like the immunoglobulin-induced remyelination in the TMEV model of MS. The timing of the onset of clinical improvement (one to two months) was similar to the time course for spontaneous remyelination ${ }^{22-24}$ and for antibody-mediated remyelination. ${ }^{20}$ Alternatively, clinical improvement after IVIg may have occurred without morphological evidence of remyelination by an effect on optic nerve conductance. After demyelination, conduction can be restored by remyelination and by the re-establishment of saltatory conduction. Another possible mechanism of axon recovery after demyelination is continuous conduction. IVIg may have induced the capacity for optic nerves to conduct like unmyelinated fibres by the development of sodium channels in the internodal area. Thus IVIg may have stimulated a spontaneously occurring adaptive repair mechanism in demyelinated fibres which has been reported in experimental demyelination and in patients with MS. ${ }^{25}$ We cannot exclude this mechanism for improving visual acuity in the reported patients. However, the timing of the onset of clinical improvement (one to two months) was similar to the time course for

Intravenous polyclonal IgG promotes CNS remyelination in TMEV-infected mice

\begin{tabular}{|c|c|c|c|c|}
\hline \multirow[b]{3}{*}{ Treatment } & \multirow[b]{3}{*}{$N \ddagger$} & \multicolumn{3}{|c|}{ CNS Remyelination } \\
\hline & & \multirow[b]{2}{*}{$\begin{array}{l}\text { No } \\
\text { Mean (SD) }\end{array}$} & \multirow[b]{2}{*}{$\begin{array}{l}\text { Area }\left(m^{2} m^{2}\right. \\
\text { Mean }(S D)\end{array}$} & \multirow{2}{*}{$\begin{array}{l}\% \text { Area CNS Remyel } \\
\begin{array}{l}\text { Area CNS Demyel } \\
\text { Mean (SD) }\end{array}\end{array}$} \\
\hline & & & & \\
\hline $\begin{array}{l}\text { Mouse IgG (iv) } \\
\text { BBS (iv) } \dagger\end{array}$ & $\begin{array}{l}7 \\
7\end{array}$ & $\begin{array}{r}11 \cdot 4(7 \cdot 6) \\
4 \cdot 3(2 \cdot 4)\end{array}$ & $\begin{array}{l}0.37(0.30) \\
0.06(0.04)\end{array}$ & $\begin{array}{c}21 \cdot 4(19 \cdot 1) \oint \\
6 \cdot 2(4 \cdot 5)\end{array}$ \\
\hline
\end{tabular}

spontaneous remyelination ${ }^{22-24}$ and for antibody-mediated remyelination, ${ }^{20}$ which suggests clinical improvement by remyelination.

The possible role of IVIg in restoring clinical function and promoting CNS remyelination needs to be confirmed with a larger, controlled trial. This pilot study was performed to determine if a more extended controlled and "blinded" study would be worthwhile. A controlled trial testing immunoglobulin in MS patients with severe functional neurological deficits is approved and underway at the Mayo Clinic.

\section{Pooled immunoglobulins from normal non-syngeneic mice promotes CNS remyelination in the Theiler's model of MS}

To further bridge the gap between the experimental studies and this pilot study on IVIg in chronic optic neuritis, we studied the effect of commercially available polyclonal IgG (Sigma Co, St Louis, MO) prepared from nonsyngeneic donor mice on TMEV-induced demyelination in SJL/J mice. The effect on remyelination by this product (dose $0.1 \mathrm{mg}$ each week over five weeks administered intravenously) which is analogous to the human IVIg preparation, was measured by quantitative morphometry as described earlier. ${ }^{20}$ For controls we used SJL/J mice treated with borate buffered saline. There were three times more lesions with remyelination in the polyclonal IgG-treated group than in mice receiving control treatment (table). In addition, the total area of CNS-type remyelination in mice receiving polyclonal IgG was six times that of the control mice. The difference between the polyclonal IgG group and the control group in the percentage of demyelinated area that showed remyelination was statistically significant $(\mathrm{p}<0.05)$. The extent of CNS remyelination observed following treatment with normal polyclonal IgG was similar to the extent of remyelination observed following treatment with CNS-specific immunoglobulins. ${ }^{2021}$ If the mechanism of remyelination is mediated by CNS-specific autoantibodies, then this would imply that these autoantibodies were present in the normal polyclonal commercially available IgG preparation, as has been shown by others for IgG isolated from pools of normal sera from various mouse strains. ${ }^{26}$

Despite the differences in route and dose, pooled polyclonal IgG from normal non-syngeneic mice promoted remyelination, while this was not the case in mice receiving IgG obtained from syngeneic SJL/J mice immunised with incomplete Freunds adjuvant or mice immunised with phosphate buffered saline. ${ }^{20} \mathrm{We}$ propose that there is a broader repertoire of antibodies (some with remyelinating potential) in the pooled polyclonal IgG from normal mice than in the laboratory-prepared IgG from the inbred strain of SJLJ mice.

In conclusion, these data show that polyclonal, commercially-available mouse immunoglobulin from non-syngeneic mice promotes 
remyelination in a viral model of MS. These findings increase the likelihood that human IVIg (non-syngeneic with multiple specificities) may also promote remyelination in human demyelinating diseases like MS.

\section{The physiological role of myelin in} demyelination and remyelination

If factors in the humoral immune system are shown to induce remyelination in MS and experimental models of demyelination, the question remains whether remyelination is necessary and sufficient for clinical recovery. Is there a one-to-one relationship between myelin and clinical symptoms? Does loss of myelin cause clinical symptoms, and does repair of myelin reduce them?

In MS, many demyelinating lesions observed by MRI and by pathological analysis remain asymptomatic early in the course of the disease. Later in the disease course there is no clear correlation between clinical symptoms and MRI abnormalities. ${ }^{27}$ If demyelination is necessary and sufficient for the development of clinical deficits in MS, then it has been inferred that the absence of neurological deficits in patients with multiple MRI lesions is the result of strategically-placed lesions in clinically "silent" areas of the CNS. Another possible explanation is that demyelination is not sufficient to cause disease.

Compared with TMEV infection of immunocompetent SJL/J mice which shows both CNS demyelination and inflammation, the toxic model of lysolecithin which induces a relatively pure demyelinated lesion without inflammation, does not produce observable clinical symptoms in the mouse. Admittedly, this lesion is usually relatively small, but can be strategically-placed. In addition, TMEV infection of mice that have the $\beta_{2}$-microglobulin gene disrupted, and therefore do not express MHC class I molecules or CD8+ $\mathrm{T}$ cells, ${ }^{28}$ indicates that demyelination is necessary but not sufficient for the development of neurological deficits. TMEV-infected $\beta_{2}$ mutant mice develop, in contrast to other susceptible strains of mice, prominent multifocal histological demyelination without significant lymphocytic inflammatory responses and without clinical signs. This suggests that in the absence of inflammation, or at least a significant alteration in the extracellular milieu, demyelination is not sufficient to produce clinical symptoms. Therefore, the isolated pathophysiological role of myelin in demyelinating disease must be reconsidered.

\section{The significance of IVIg administration for the concepts "immunoglobulin" and "myelin"}

The clinical and laboratory experiments to enhance remyelination with IVIg administration enables us to question two fundamental concepts: (1) the role of immunoglobulin in MS, and (2) the physiological role of myelin in conditions of demyelination and remyelination.

The concept "immunoglobulin" in MS

The availability, suggested therapeutic efficacy, and safety of IVIg treatment in humans, and the antibody-mediated remyelination in experimental animals has encouraged clinicians and immunologists to reassess the role of immunoglobulin in autoimmune diseases. ${ }^{89192930}$ The specificity of the oligoclonal immunoglobulin in cerebrospinal fluid and MS lesions remains undetermined; their pathogenicity has not been proved despite extensive investigation. ${ }^{31}$ In tissue cultures, the major demyelinating factor of MS serum seems to be a component other than IgG. ${ }^{32}$ Intrathecal IgG synthesis occurs in patients with other neurological disease (even in normal subjects) and no correlation exists between levels of intrathecal IgG synthesis and the progression of MS. ${ }^{33-35}$ Direct transfer of intrathecal IgG from MS patients or from animals with experimental allergic encephalomyelitis (EAE) fails to induce demyelination in recipient animals. ${ }^{31}$ In contrast, immunisation with myelin components in animals with EAE have been shown to induce oligodendroglia proliferation, and to inhibit the development of EAE. ${ }^{36}$ From these experimental and clinical data the traditional notion of the immune response (in particular IgG) solely as deleterious involved in the pathogenesis of demyelinating diseases like MS should be reconsidered.

Intrathecal IgG synthesis may function as a marker of a stimulated immune response, or may represent an immune response secondary to myelin breakdown playing a physiological role in the catabolism of endogenous substances. ${ }^{37}$ A certain proportion of the oligoclonal cerebrospinal fluid IgG in MS may belong to the large family of naturally occurring autoantibodies, ${ }^{35}$ and may be involved in promoting CNS remyelination similar to the autoreactive antibodies (autoantibodies against spinal cord homogenate) in the TMEV model of demyelination. ${ }^{1920}$ The elevated IgG, therefore, may reflect an attempt at immunological homeostasis. Correspondingly, and according to these ideas, autoimmune diseases, and possibly also MS may be treated successfully not by suppressing the immune network, but by strengthening it by administration of IVIg. This suggestion agrees with the frequent association of autoimmunity with immunodeficiency. ${ }^{30}$

\section{The concept "myelin" in demyelination} and remyelination

Because CNS demyelination appears not to be sufficient to cause symptoms either in humans or in the experimental models, we propose a double-hit mechanism in the pathophysiology of MS. The first hit causes injury to myelin and/or oligodendrocytes resulting in denuded axons. This may be 
induced by various factors including viruses and toxins, or by autoimmune mechanisms directed against oligodendrocytes or the myelin sheath. Once the axon is naked, then it is vulnerable for the second hit that could cause a chronic axonopathy with prominent clinical symptoms. ${ }^{38}$ This subsequent damage may be elicited non-specifically by immune cells, cytokines, or other factors that are recruited to the lesion.

Conforming to this hypothetical double-hit model, myelin may have a second function besides an electrical insulator, that of a protective barrier for the axon. In this view, remyelination may prevent an axonopathy indirectly. Demyelination alone is not sufficient to cause symptoms, but immunoglobulin-mediated remyelination may reduce or prevent an otherwise irrecoverable clinical deficit by protecting the naked axon membrane from subsequent immune attacks. Remyelination may prohibit the development of a chronic axonopathy and Wallerian degeneration. IVIg treatment in demyelinating diseases such as MS is not the final treatment, but represents a stage in the search for more specific remyelination-promoting immune factors.

This study was supported by a grant ( $92-115 \mathrm{~ms}$; to BvE) of the Dutch Society for Support of Research on Multiple
Sclerosis, and by a grant (NS 24180) from the National Institute of Health, USA.

1 Goodkin DE, Ransohoff RM, Rudick RA. Experimental herapies for multiple sclerosis: current status. Clev Clin therapies for multiple

2 Miller HG, Foster JB, Newell DJ, Barwick DD, Brewi RAL. Multiple sclerosis: therapeutic trials of chloroquine, soluble aspirin, and gammaglobulin. BMF 1963 1:1436-9.

3 Schuller E, Govaerts A. First results of immunotherapy with immunoglobulin $G$ in multiple sclerosis patients. Eur Neurol 1983;22:205-12.

4 Soukop W, Tschabitscher H. Gammaglobulintherapie bei multipler sclerose (MS): Theoretische Uberlegungen und erste klinische Erfahrungen mit 7S-Immunoglobulin in der MS-Therapie. Wien Med Wschr 1986; globulin in

5 Yan J, Richert JR, Sirdofsky MD. High-dose intravenous immunoglobulin for multiple sclerosis. Lancet 1990;2: immun.

6 Achiron A, Pras E, Gilad R, et al. Open controlled therapeutic trial of intravenous immune globulin in relapsing-remitting multiple sclerosis. Arch Neurol 1992 49:1233-47.

7 Cook SD, Troiano R, Rohowsky-Kochan C, et al. Intravenous gamma globulin in progressive multiple sclerosis. Acta Neurol Scand 1992;86:171-5.

8 van Engelen BGM, Hommes OR, Pinckers A, et al. Improved vision after intravenous immunoglobulin in stable demyelinating optic neuritis. Ann Neurol 1992; 32:835-36.

9 Tenser RB, Hay KA, Aberg JA. Immunoglobulin G immunosuppression of multiple sclerosis, suppression of all three major lymphocyte subsets. Arch Neurol 1993; 50:417-20.

10 Miller DJ, Rodriguez M. Spontaneous and induced remyelination in multiple sclerosis and the Theiler' virus model of central nervous system demyelination. Microscopy Res and Technique (in press).
11 Prineas JW, Barnard RO, Kwon EE, et al. Multiple sclerosis: remyelination of nascent lesions. Ann Neurol 1993;33:137-151.

12 Rodriguez M, Scheithauer BW, Forbes G, Kelley PJ Oligodendrocyte injury is an early event in lesions of multiple sclerosis. Mayo Clin Proc 1993;68:627-36.

13 Rodriguez M, Scheithauer BW. The ultrastructure of multiple sclerosis. Ultrastruct Pathol 1994;18:3-13.

14 Greenfield JG. Demyelinating diseases. In: JG Greenfield, ed. Neuropathology London: Edward Arnold, 1958 441-74.

15 Kerlero de Rosbo N, Honegger P, Lassmann H, Matthieu JM. Demyelination induced in aggregating brain cell cultures by a monoclonal antibody against myelin oligodendrocyte glycoprotein. $₹$ Neurochem 1990;55:583-7.

16 Mastaglia FL, Carroll WM, Jennings AR. Spinal cord lesions induced by antigalactocerebroside serum. Clin Exp Neurol 1989;26:33-44.

17 Raine CS, Diaz M, Pakingan M, Bomstein MB. Antiserum induced dissociation of myelinogenesis in vitro: an ultrastructural study. Lab Invest 1978;38:397-403.

18 Bansal R, Gard AL, Pfeiffer SE. Stimulation of oligodendrocyte differentiation in culture by growth in the presence of a monoclonal antibody to sulfated glycolipid f Neurosci Res 1988;21:260-67.

19 Rodriguez M, Lennon VA, Benveniste EN, Merrill JE. Remyelination by oligodendrocytes stimulated by antiserum to spinal cord. 7 Neuropath Exp Neurol 1987;46: 84-95.

20 Rodriguez $M$, Lennon VA. Immunoglobulins promote remyelination in the central nervous system. Ann Neurol 1990;27:12-17.

21 Rodriguez M. Immunoglobulins stimulate central nervous system remyelination: electron microscopic and 1991;64:358-70.

22 Blakemore WF. Demyelination in the superior cerebellar peduncle in the mouse induced by Cuprizone. $\mathcal{f}$ Neurol Sci 1973a;20:63-72.

23 Blakemore WF. Remyelination of the superior cerebellar peduncle in the mouse following demyelination induced by feeding Cuprizone. $\mathcal{F}$ Neurol Sci 1973;20:73-83.

24 Ludwin SK. Remyelination in the central nervous system and the peripheral nervous system. Adv Neurol 1988 47:215-54.

25 Moll C, Mourre C, Lazdunski M, Ulrich J. Increase of sodium channels in demyelinated lesions of multiple sclerosis. Brain Res 1991;556:311-16.

26 Berneman A, Ternynck T, Avramas S. Natural mouse IgG reacts with self antigens including molecules IgG reacts with self antigens including molecules involved in

27 Wiebe S, Lee DH, Karlik SJ, et al. Serial cranial and spinal cord magnetic resonance imaging in multiple sclerosis. Ann Neurol 1992;32:643-50.

28 Rodriguez M, Dunkel AJ, Thiemann RT, et al. Abrogation of resistance to Theiler's virus-induced demyelination in $\mathrm{H}-2 \mathrm{~b}$ mice deficient in beta2-microglobulin Immunol 1993;151:266-76.

29 Varela FJ, Coutinho A. Second generation immune networks. Immunol Today 1991;12:159-66.

30 Coutinho A. Beyond clonal selection and network. Immunol Review 1989;110:63-87.

31 Trotter $J \mathrm{~L}$, van der Veen RC. Autoantibodies in multiple sclerosis. In: Cook SD, ed. Handbook of multiple sclerosis. New York: Marcel Dekker, 1990:151-68.

32 Grundke-Iqbal I, Bornstein MB. Multiple sclerosis: serum gamma globulin and demyelination in organ culture. Neurol 1980;30:749-54.

33 Rostrom B. Specificity of antibodies in oligoclonal bands in patients with multiple sclerosis and cerebrovascular disease. Acta Neurol Scand 1981;63:1-84.

34 Walsh MJ, Tourtelotte WW. Temporal invariance and clonal uniformity of brain and CSF IgG, IgA, and IgM in multiple sclerosis. $\mathcal{F}$ Exp Med 1986;163:41-53.

35 Matsiota P, Blancher A, Doyon B, et al. Comparative study of natural autoantibodies in the serum and cerebrospinal fluid of normal individuals and patients with multiple sclerosis and other neurological diseases. Ann Immunol (Inst Pasteur) 1988;139:99-108.

36 Raine CS, Moore GRW, Hintzen R, Traugott U. Induction of oligodendrocyte proliferation and remyelination after chronic demyelination. Lab Invest 1988; nation after

37 Grabar P. Autoantibodies and the physiological role of immunoglobulins. Immunol Today 1983;4:337-40.

38 Barnes D, Munro PMG, Youl BD, et al. The longstanding MS lesion quantitative MRI and electron microscopic study. Brain 1991;14:1271-80. 